\title{
Therapie akuter Intoxikationen
}

\author{
Carsten Hafer, Jan T. Kielstein
}

Intoxikationen geben ein sehr unterschiedliches und meist nicht eindeutiges klinisches Bild ab. Toxidrome erleichtern die korrekte Diagnosestellung und die zumindest grobe Zuordnung zu einer Substanzgruppe - Voraussetzung einer zielgerichteten Therapie.

\section{Epidemiologie}

Intoxikationen sind bei Jugendlichen die dritthäufigste Todesursache und zählen auch bei jüngeren Erwachsenen zu den häufigeren Todesursachen. Etwa 1-2\% der stationären Behandlungen und rund 5-10\% aller Notarzteinsätze sind auf akute Intoxikationen zurückzuführen.

Grundsätzlich unterscheidet man akzidentielle von vorsätzlichen Vergiftungen, wobei Letztere oft Folge von Suizidversuchen sind und mit der Einnahme von Analgetika (v.a. Paracetamol), Sedativa und Hypnotika (Benzodiazepine) sowie Psychopharmaka (v.a. Antidepressiva) in Zusammenhang stehen - meist als Mischintoxikation [1,2].

Die Mortalität ist erfreulicherweise relativ gering und beträgt weniger als $1 \%$. Dennoch starben laut Bundeskriminalamt 2011 in Deutschland 10144 Menschen durch Suizid. Die Zahl der Suizidversuche liegt deutlich höher und beträgt Schätzungen zufolge mehr als das 10-Fache. Bei einem Großteil der Suizidversuche liegt eine Vergiftung zugrunde. Beim überwiegenden Teil akuter Intoxikationen handelt es sich um die Volksdroge Alkohol (ca. 63\%), weitere häufige Fälle betreffen Drogenintoxikationen. Hierbei werden die Klassiker Kokain, Heroin und Cannabinoide zunehmend durch moderne Designerdrogen wie „Badesalze“ abgelöst. Diese Produkte werden im Internet unter verschiedenen Namen angeboten und erhalten häufig Rauschmittel, Stimulanzien oder ähnliche chemische Wirkstoffe, die auf den Verpackungen nicht ausgewiesen sind (z.B. Mephedron) und die zum Teil auch nicht bekannt oder nur sehr schwer zu identifizieren sind [3].

Unfälle mit Brandbeschleunigern, Haushaltsreinigern sowie die versehentliche Einnahme von Zigaretten, Pflanzen, Farben oder Tabletten spielen eine nur geringere Rolle [4] und werden in diesem Artikel auch nicht weiter behandelt.

\section{Allgemeiner Ansatz}

Bei der Behandlung von Intoxikationen können wir uns nur auf wenige kontrollierte randomisierte Studien stützen. Es liegt in der Natur dieses medizinischen Problems, dass sich dies auch nicht ändern wird. Somit sollten allgemeinen Therapieansätzen immer spezifische Behandlungen folgen, die auf den toxikologischen Eigenschaften und den neusten fallbasierten Erkenntnissen beruhen. 


\section{Infobox 1}

Orientierende Regeln zum Vorgehen bei Intoxikationen
5-Fingerregel bei Intoxikationen:

- lebensrettende Basismaßnahmen

- Giftelimination

- Antidottherapie

- Asservierung von Proben

- Transport

6 W-Fragen:

- Wer (Alter, Gewicht des Betroffenen)
- Was (genauer Name des Giftstoffs bzw. Produkts, am besten von der Packung ablesen)

- Wann (genauer Einnahmezeitpunkt)

- Welche Menge (genaue Mengenangabe bzw. maximal mögliche Menge)

- Was wurde bisher unternommen?

- Wie geht es dem Patienten?
Wie in jeder anderen Notfallsituation ist ein systematischer und einheitlicher Ansatz zur Primärtherapie hilfreich - am besten anhand fester Handlungsanweisungen. Er erspart insbesondere bezüglich der Probenasservierung und sonstigen Analytik viel Zeit. $\mathrm{Zu}$ beachten ist ferner, dass Intoxikationen - insbesondere Mischintoxikationen - eine sehr breite Palette an Symptomen und klinischen Befunden bieten, wovon nur wenige spezifisch sind und direkt auf die aufgenommene Substanz hinweisen. Die wesentlichsten Faktoren für die Schwere einer Vergiftung sind die Giftmenge und die Zeitdauer seit der Einnahme.

Paracelsus hat die wichtigste toxikologische Aussage bereits vor knapp 500 Jahren gemacht: „Die Dosis macht das Gift“. Ergänzend müsste nur noch die Zeitdauer des Einwirkens hinzugefügt werden.

Schwere einer Vergiftung $=$ Giftmenge $\times$ Zeit .

Die Behandlung selbst besteht bei schweren Vergiftungen in Ermangelung konkreter Hinweise auf eine optimale Versorgung meist wie bei anderen Intensivpatienten auch aus supportiven Maßnahmen. Ergänzt werden sie durch Maßnahmen zur Absorptionsminderung und Giftentfernung sowie - falls möglich - die Gabe von Antidota [5]. Neben den klassischen schnell wirksamen Antidota sind aber auch frühzeitige andere Therapieformen zu diskutieren, z. B. bei einer Paraquatintoxikation eine Chemotherapie und Immunsuppression, um die drohende Lungenfibrose zu minimieren oder zu verhindern [6].

Eine vitale Bedrohung ist bei fehlenden oder geringen Symptomen und unklarer Latenzzeit nicht auszuschließen. Eine medizinische Überwachung ist so lange erforderlich, bis die laboranalytische und medizinische Diagnostik eine schwere Vergiftung ausschließen kann.

Generell kann man sich an den 6 W-Fragen und der 5-Finger-Regel orientieren (Infobox 1).

\section{Diagnostik}

\section{Umgebung und Begleitumstände}

Ergänzende Hinweise zu den anamnestischen Angaben findet man häufig bei der Inspektion der Umgebung, in der der Patient aufgefunden wird. Hierbei erhält man evtl. Hinweise auf ingestierte Substanzen durch Tablettenblister oder auf dem Boden liegende Tabletten sowie auch auf die Begleitumstände (Abschiedsbrief als Hinweis auf Suizid). Gerade beim suizidalen, unkooperativen oder vigilanzgeminderten Patienten ist es unerlässlich, die unmittelbare Umgebung des Patienten genau zu inspizieren. Mit detektivischer Arbeit darf man jedoch immer erst nach vitaler Stabilisierung des Patienten beginnen. Das Hauptaugenmerk gilt Medikamenten, Alkohol und Hilfsmitteln zur Rauschmittelaufnahme (z. B. Injektionsnadeln, ggf. mit Restbeständen). Eine Asservierung von Erbrochenem oder anderen Körperflüssigkeiten für spätere toxikologische Analysen hat nur selten einen unmittelbaren klinischen Nutzen, da meist andere Befunde früher vorliegen oder die Behandlung im Vordergrund steht. Es ist aber niemals falsch, diese Asservate zu sichern wegwerfen kann man sie später immer noch.

\section{Anamnese}

Man muss unterscheiden, ob ein akuter oder chronischer Missbrauch einer toxischen Substanz vorliegt. Vorbestehende medikamentöse Verschreibungen sind hinsichtlich der Potenzierung und Wechselwirkungen ebenso wichtig wie das Erfassen rezeptfreier Präparate oder anderer Begleitsubstanzen (z. B. Öle, Haushaltsreiniger, Kühlmittel). All diese Informationen sind wichtig für weitere differenzialtherapeutische Überlegungen. In diesem Zusammenhang ist erwähnenswert, dass man den Angaben einer bewusst intoxikierten Person nicht allzu viel Glauben schenken sollte. Nur bei etwa einem Viertel bis maximal der Hälfte der Fälle stimmen die Angaben mit den tatsächlichen Befunden überein [7,8]. Keinesfalls sollte man jedoch die Anamnese vernachlässigen, vielmehr muss man die Angaben mit den Symptomen korrelieren. Zudem sollte man die Informationen möglichst durch fremdanamnestische An- 
gaben vertiefen (Polizei, Rettungsdienst, Angehörige, neugierige Nachbarn, Arbeitskollegen, Ex-Freundin).

\section{Körperliche Untersuchung}

Intoxikationen können sich mit einer Vielzahl klinischer Beschwerden präsentieren, von abdominellen Schmerzen und Erbrechen über neurologische Auffälligkeiten wie Tremor, Bewusstseinseinschränkungen oder Krampfanfälle bis zu respiratorischen Problemen und Rhythmusstörungen. Die körperliche Untersuchung ist daher in ihrer Bedeutung nicht zu unterschätzen. Sie liefert die ersten wesentlichen Hinweise auf ingestierte Substanzen, die für die einzuleitenden therapeutischen Schritte wesentlich sind. Klassische Veränderungen sind Pupillenveränderungen wie eine ausgeprägte Miosis bei Opioiden oder eine Mydriasis bei Sympathomimetika.

Die toxische Wirkung einer meist ja noch unbekannten Substanz kann sich ändern. Vor allem bei Substanzen mit verzögert einsetzender Wirkung sollte man die Untersuchung engmaschig wiederholen, da sich die typische Symptomatik erst nach einer Latenzzeit einstellt.

Toxidrome sind vergiftungsbedingte Symptomenkomplexe, die man in bestimmten Fällen zur Differenzialdiagnose heranziehen kann. Daher ist die Identifizierung eines Toxidroms das Schlüsselelement bei der initialen Beurteilung eines vergifteten Patienten. Die an Toxidromen orientierte Identifizierung eines Problems bietet die Basis einer rationaleren und schnelleren Herangehensweise als die Konzentration auf ein bestimmtes vermutetes Gift.

Die körperliche Untersuchung konzentriert sich dabei auf die klassischen Vitalparameter (Blutdruck, Puls, Atemfrequenz, Temperatur, Hydratationszustand, Sauerstoffsättigung, Blutzucker) sowie auf den neurologischen Status mit Bewusstseinsgrad (wach, Reaktion auf Ansprache, auf Schmerzreize oder komatös), Muskeltonus und Pupillen (Größe, Position und Reaktion).

Anhand dieser Kriterien kann man den Patienten recht gut klassifizieren:

Physiologische Exzitation. ZNS-Stimulation, Tachykardie, erhöhter Blutdruck, gesteigerte Atemfrequenz und Temperatur:

- Anticholinergika

- Sympathomimetika

- halluzinogene Substanzen
Physiologische Depression. Gedämpfte Bewusstseinslage, niedriger Blutdruck, niedrige Herzfrequenz, niedrige Atemfrequenz:

- Alkohole, Sedativa

- Opiate

- Cholinergika (parasympathomimetisch)

- Sympatholytika

Gemischte physiologische Effekte. Mischbild aufgrund von Polytoxikomanie oder Stoffen mit einer Vielzahl von Wirkungen:

- eine Hypoglykämie induzierende Substanzen

- Salizylate

- Zyanide

- trizyklische Antidepressiva

In Ergänzung zu den genannten Symptomen sollte man auch nach Hinweisen auf Traumata, Einstichstellen oder eine transdermale Medikation (Pflaster) suchen, ferner nach Anzeichen eines Krampfanfalls (Harn- und oder Stuhlabgang, Zungenbiss). Zusätzlich liefert die Untersuchung auf vegetative Symptome (Farbe und Feuchtigkeit der Haut und Schleimhäute, Schwitzen, Speichelfluss, Darmgeräusche) und Gerüche wichtige Informationen.

\section{EKG}

Das EKG ist eine unverzichtbare und unter Umständen lebensrettende Basismaßnahme, die bei jedem intoxikierten Patienten (wiederholt) durchgeführt werden sollte. Veränderungen liefern zum Teil diagnostische, aber auch prognostisch relevante Informationen (Infobox 2) [9].

\section{Infobox 2}

Interpretation des EKG bei Intoxikation

- Rhythmus (ventrikulär vs. supraventrikulär)

- Herzfrequenz (Tachykardie, Bradykardie)

- Ektopien

- PR-Intervall: Verlängerung bei Kalzium, Digitalis, Betablockern und bei erniedrigtem Sympathikotonus

- QRS-Dauer: Verlängerung ist immer verdächtig auf Natriumkanalblockade

- QTc-Zeit: je länger, desto gefährlicher

- ST-Segmentveränderungen 
Abb. 1 Typische EKG-Befunde bei Intoxikationen. a Natriumkanalblockade: je größer die Blockade, desto breiter der QRSKomplex. b Kaliumkanalblockade: verlängertes QT-Intervall, evtl. U-Welle.
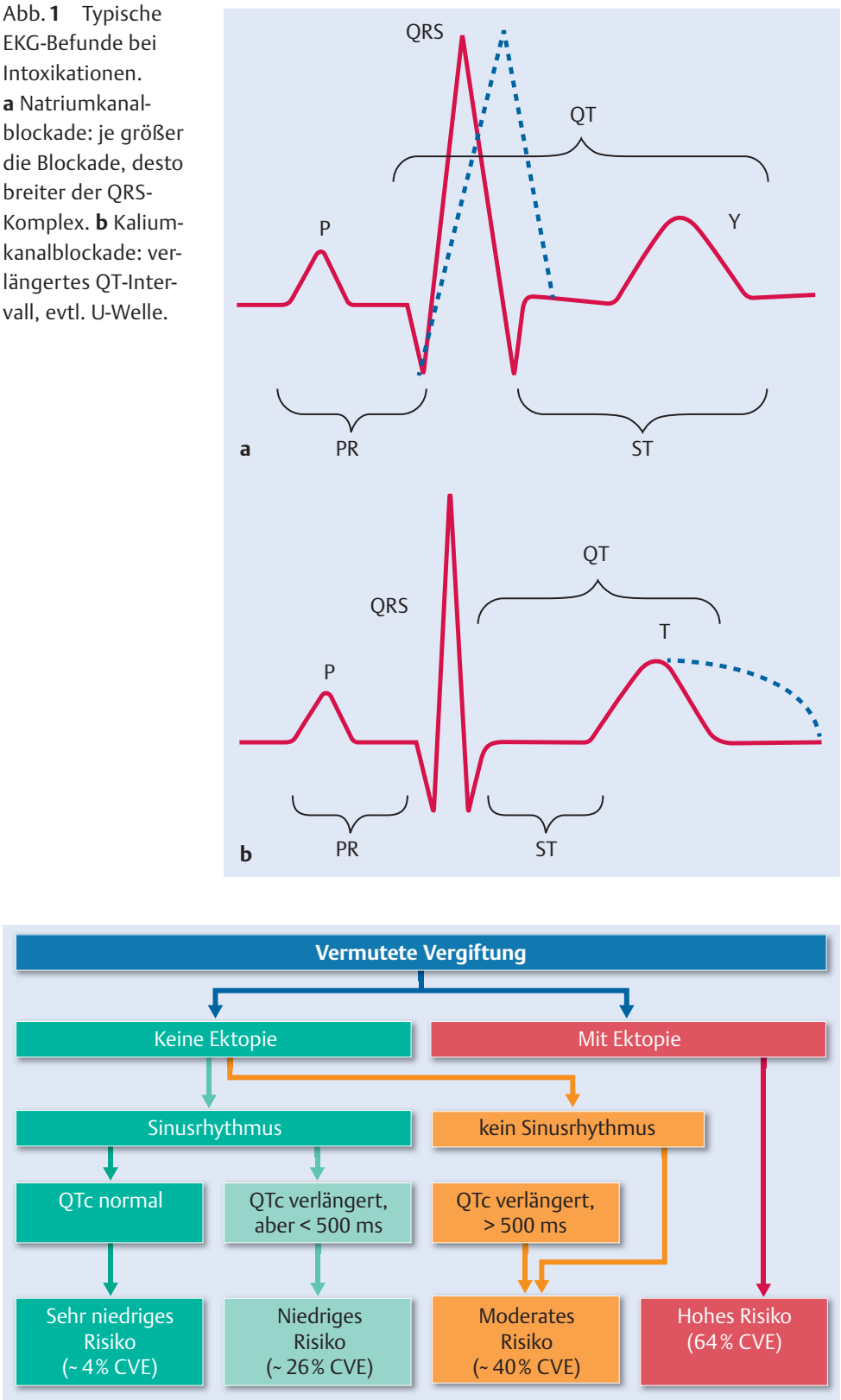

Abb. 2 Abschätzen des Risikos kardiovaskulärer Ereignisse (CVE) bei Vergiftungen anhand des EKG (mod. nach [9]). unverzügliche Natriumbikarbonatgabe. Bei Intoxikation mit trizyklischen Antidepressiva korreliert das Risiko für zerebrale Krampfanfälle oder ventrikuläre Dysrhythmien mit der maximalen QRS-Dauer in einer Extremitätenableitung [10].

Von keinem nichtkardiologischen Intensivmediziner kann erwartet werden, alle potenziell bedrohlichen Rhythmusstörungen oder subtilen Veränderungen eindeutig und sofort zu erkennen. Tatsächlich fanden sich bei der Hälfte der initialen EKG-Beurteilungen Fehlinterpretationen, die andere Handlungsempfehlungen der Giftzentrale nach sich gezogen hätten [11].

Es ist beim EKG sehr hilfreich, sich eines EKG-Toxidroms oder eines einfachen Algorithmus' zu bedienen (Abb.2).Zudem sollte man sich angesichts der enormen Bedeutung nicht scheuen, ein „suspektes“ EKG einem Experten zu zeigen.

Überwachung. Ein Großteil intoxikierter Patienten sollte aus vitalen und nicht zuletzt auch forensischen Gründen bei fortbestehender Suizidalität am Monitor überwacht werden und belegt daher wichtige intensivmedizinische Kapazitäten. Da eine vitale Bedrohung bei fehlenden oder geringen Symptomen und unklarer Latenzzeit nie auszuschließen ist, überwacht man den Patienten meist über 24 Stunden am Monitor (Infobox 3). Bei Patienten mit einem normalen Sinusrhythmus, keinerlei Hinweisen für eine Kardiotoxizität und normalem körperlichem Untersuchungsbefund genügt aber wahrscheinlich ein Beobachtungszeitraum von 6-8 Stunden.

Die Gefahr einer Vergiftung sind nicht die zentralen Wirkungen, denn Schweregrad und Bewusstseinslage korrelieren nur schlecht bis gar nicht. Bedrohlich sind vielmehr Rhythmusstörungen, Krampfanfälle und pulmonales Versagen infolge von Aspiration und gestörter Atemfunktion.

\section{Infobox 3}

Kriterien für eine intensivmedizinische Überwachung

- arterieller $\mathrm{CO}_{2}$-Partialdruck $\left(\mathrm{p}_{\mathrm{a}} \mathrm{CO}_{2}\right)>45 \mathrm{mmHg}$

- Notwendigkeit der endotrachealen Intubation

- Krampfanfall

- keine Reaktion auf Ansprache

- kein Sinusrhythmus

- zweit- oder drittgradiger AV-Block

- QRS-Dauer $\geq 0,12 \mathrm{~s}$

- systolischer Blutdruck unter $80 \mathrm{mmHg}$ 


\section{Labor}

Oft sind in der Notfallmedizin Patienten mit akuten Symptomen zu behandeln, für die es zunächst keine naheliegende Erklärung gibt. Im Laufe der Diagnostik lassen mitunter pathologische Untersuchungs- und Laborwerte an die Möglichkeit einer Vergiftung denken.

Routinelabor. Generell sollte man bei der stationären Aufnahme vergifteter Personen ein umfangreiches Routinelabor abnehmen. Hinweise auf mögliche sekundäre Organschädigungen wie eine Einschränkung der Nierenfunktion, Zeichen der Hepatotoxizität oder Zeichen einer Rhabdomyolyse lassen sich so frühzeitig und vor allem im weiteren Verlauf richtig einordnen. Ein Differenzialblutbild und eine Blutgerinnung sind ebenfalls wichtig. Sofern möglich sollte man diese Befunde mit Vorbefunden vergleichen (beim Hausarzt anfordern), um ggf. akute von chronischen Veränderungen zu unterscheiden.

Säure-Basen-Haushalt. Essenziell (aus Sicht des Autors die wichtigste Untersuchung überhaupt) ist bei allen Intoxikationen die - ggf. wiederholte - Bestimmung des Säure-Basen-Haushalts und der Serumelektrolyte Natrium, Kalium und Chlorid. Zum einen hilft die Errechnung der Anionenlücke differenzialdiagnostisch weiter (Infobox 4), andererseits kann man damit die Dynamik des Krankheitsgeschehens (cave: progredienter Bikarbonatabfall) und eine respiratorische Erschöpfung $\left(\mathrm{CO}_{2}\right.$-Anstieg) erkennen.

Die BGA sollte man bei instabilen Patienten zur Therapie- und Verlaufskontrolle regelmäßig und engmaschig (stündlich) kontrollieren.

Eine metabolische Azidose mit erhöhter Anionenlücke ist ein Hinweis auf exogen zugeführte oder endogen entstandene Säuren. Exogen zugeführte Säuren sind bei Intoxikationsverdacht in erster Linie Salizylate und die toxischen Alkohole Methanol und Ethylenglykol [12], aber leider auch noch eine ganze Reihe weiterer Substanzen.

Endogen entstehende Säuren sind in erster Linie Laktat- (auch an Metformin denken) und Ketoazidosen (Kontrolle im Urinstatus auf Ketonurie). Zur weiteren Differenzierung helfen die Serumosmolalität und die Berechnung der osmotischen Lücke (Infobox 4).

\section{Infobox 4}

Wichtige Laboruntersuchungen bei Intoxikationen
- Routinelabor

- Differenzialblutbild

- Gerinnungsstatus

- Blutgasanalyse (ggf. stündlich)

- Elektrolyte (ionisiertes Kalzium, ionisiertes Phosphat, Natrium, Kalium, Chlorid)

- Laktat

- Urinstatus
Berechnung der Anionenlücke: Anionenlücke $=\mathrm{Na}^{+}-\left(\mathrm{HCO}_{3}{ }^{-}+\mathrm{Cl}^{-}\right)$ Berechnung der Osmolalität: errechnete Osmolalität $=$ $2 \times$ Natrium + Harnstoff + Glukose (alle in $\mathrm{mmol} / \mathrm{l}$ ) Berechnung der osmotischen Lücke: bestimmte Osmolalität-errechnete Osmolalität
Blutgasanalyse. Eine arterielle (zumindest kapilläre) Blutgasanalyse ist bei allen respiratorisch eingeschränkten Patienten notwendig. Bei Patienten im Schock sind die gleichzeitige Abnahme von arterieller und venöser BGA und die Bestimmung der arteriovenösen Sauerstoffdifferenz hilfreich. Liegt eine Rauchgasintoxikation vor, sollte man auch Kohlenmonoxid und Methämoglobin bestimmen (letzteres auch bei allen Zyanidintoxikationen).

Weitere Parameter. Initial sollte man zudem den Kalzium- und Phosphatspiegel bestimmen, da Hypophosphatämien zur Kardiotoxizität beitragen können. Beim Kalzium ist das ionisierte Kalzium als das biologisch aktive Äquivalent zu kontrollieren (wird von modernen BGA-Geräten meist automatisch bestimmt; Normalbereich: 1,1-1,4 mmol/l bzw. 4,5-5,6 mg/dl). Falls das Blutgasgerät kein Chlorid bestimmen kann, sollte man diesen Wert im Labor anfordern. Außerdem sollte man Laktat kontrollieren und einen Urinstatus erheben. Ein entsprechendes Toxidrom kann in Kombination mit einer Hyponatriämie z. B. auf eine Intoxikation mit Ecstasy hinweisen.

Schwangerschaftstest. Bei Frauen im gebärfähigen Alter ist zudem ein Schwangerschaftstest empfehlenswert.

Substanzscreening. Das Screening auf bestimmte Substanzen wie Salizylate und Paracetamol ist eindeutig sinnvoll. Bei entsprechendem Verdacht sind auch Spiegelbestimmungen schnellstmöglich zu veranlassen, zumal effektive Therapien möglich sind. Insbesondere Paracetamol findet sich immer wieder als Begleitsubstanz (bei etwa $10 \%$ aller Intoxikationen), wenngleich ein Drittel der Patienten dies verneint [13]. 
Weiterführende toxikologische Serum- und Urinanalysen. Neben dem etablierten Urinscreening auf toxische Substanzen ist bei klinisch stabilen Patienten und einer unbeabsichtigten Giftaufnahme eine weiterführende toxikologische Analyse selten sinnvoll. Hierbei sind auch die Kosten zu bedenken. Im forensischen Bereich haben sich offensichtlich auch Speichelanalysen auf Betäubungsmittel bewährt (DrugTest 5000 der Firma Dräger) $[14,15]$.

Weiterführende toxikologische Serum- und Urinanalysen sind insbesondere dann sinnvoll, wenn die Anamnese lückenhaft bleibt oder wenn sich Widersprüche aus dem klinischen Bild und der vermuteten Ingestion ergeben sowie bei absichtlichen, lebensbedrohlichen Vergiftungen mit Komplikationsrisiken.

Prinzipiell sollte man aufwendigere toxikologische Laboranalysen immer in Rücksprache mit einem Giftinformationszentrum veranlassen [16]. In der Realität vergeht jedoch viel Zeit bis zum Eintreffen dieser Befunde, die häufig sogar in externen Labors erhoben werden.

Auf keinen Fall darf man in lebensbedrohlichen Fällen mit der Therapie erst nach dem Eintreffen der Analytik beginnen. Eine leere Schachtel Paracetamol reicht aus, um eine Therapie mit Acetylcystein zu beginnen.

\section{Giftinformationszentren in Deutschland}

Bei allen instabilen Patienten und bei Unsicherheiten hinsichtlich der klinischen Einordnung einer Symptomatik sollte man Kontakt mit einem Giftinformationszentrum (GIZ) aufnehmen. Diese sind in Deutschland einheitlich unter der regionalen Vorwahl und der Rufnummer 19240 erreichbar (also z. B. Berlin: 03019240 - ärztliche Vergiftungsberatung rund um die Uhr). Insbesondere zu Fragen der Asservierung und der weiteren Analytik werden hier Fragen sehr gezielt und konkret beantwortet.

\section{Suizidalität}

Jede Suizidäußerung ist ernst zu nehmen - eine ausführliche fachärztliche Exploration ist zwingend notwendig.
Zur Beurteilung der Suizidalität gehört natürlich eine ausführliche Anamnese. Suizidale Patienten benötigen eine Intensivierung des zeitlichen Engagements und der therapeutischen Bindung. Die Suizidalität ist im Gespräch offen und präzise zu thematisieren. Wichtig ist es in dieser Hinsicht auch, die Absprachefähigkeit des Patienten zu beurteilen. Akut suizidale Patienten, die nicht absprachefähig sind, sind nach somatischer Stabilisierung unverzüglich in Begleitung in eine psychiatrische Klinik einzuweisen. Bis dahin sind sie intensiv-/sitzwachenpflichtig (Infobox 5).

Alle Patienten mit einer absichtlichen Vergiftung benötigen eine psychiatrische Beurteilung, bevor man sie entlassen kann.

\section{Infobox 5}

Indikationen zur stationären Aufnahme (nach S3-Leitlinie)

- akute Suizidgefährdung, Versorgungsnotwendigkeit nach einem Suizidversuch

- Unsicherheit bei der Einschätzung der Suizidgefahr

- keine tragfähige therapeutische Beziehung

- Weiterbestehen von Suizidalität trotz adäquater Initialbehandlung

\section{Therapie}

Das Potenzial der Gefahr einer Vergiftung kann nicht auf dem Boden der Vigilanz beurteilt werden, denn Schweregrad und Bewusstseinslage korrelieren nicht gut miteinander. Die wichtigsten Gefahren sind vielmehr:

- Rhythmusstörungen

- zerebrale Krampfanfälle

- pulmonales Versagen infolge von Aspiration und gestörter Atemfunktion

\section{Basismaßnahmen}

Die Behandlung von Intoxikationen beginnt immer schon durch den Notarzt mit der Sicherung der Vitalfunktionen.

Grundprinzip: Symptomatische Therapie vor spezifischer Behandlung. 
Grundsätzlich ähnelt die symptomatische Therapie beim vergifteten Patienten der des „gewöhnlichen“ Intensivpatienten. Bei den meisten Patienten genügen alleinige symptomatischen Maßnahmen. Einige Besonderheiten, die im Folgenden genannt werden, verdienen jedoch besondere Beachtung.

Atemwegsicherung. Die Indikation zur Atemwegsicherung durch eine endotracheale Intubation sollte man bei eingeschränkter Bewusstseinslage großzügig stellen, sofern die Ursachen dafür nicht rasch reversibel sind (z. B. Hypoglykämie oder antagonisierbare Opioidintoxikation). Das Risiko einer Aspiration und damit einhergehend auch der Mortalität sind bei intoxikierten Patienten deutlich erhöht [17]. Bei einer notwendigen Intubation sollte man die Narkose als „rapid sequence induction“ mit einem Relaxans mit schneller Anschlagszeit durchführen.

Die Gefahr einer Aspiration ist beim intoxikierten Patienten besonders hoch. Eine frühzeitige Sicherung der Atemwege durch endotracheale Intubation sollte man bevorzugt erwägen.

Ausnahme davon ist eine gesicherte oder vermutete Salizylatintoxikation, bei der man eine Intubation möglichst umgehen sollte, denn hierbei besteht üblicherweise parallel eine metabolische Azidose (mit hoher Anionenlücke) und eine ausgeprägte respiratorische Alkalose. Kommt es bei der Intubation zu einer passageren Hypoventilation, entwickelt sich rasch eine profunde Azidose, die die Toxizität von Salizylaten weiter verstärkt. Daher ist in dieser speziellen Situation eine passagere Hyperventilation mit ggf. höheren Atemzugvolumina hilfreich und zu vertreten.

Die Gabe von Sauerstoff sollte generell liberal gehandhabt werden. Die Indikation zu einer antiinfektiösen Therapie sollte man bei Patienten, die in Erbrochenem aufgefunden werden, ebenfalls großzügig stellen.

Hypotonie. Zur Therapie einer Hypotonie setzt man außer bei Zeichen eines kardiogenen Schocks - initial isotone kristalloide (Vollelektrolyt-) Lösungen ein. Bei unzureichendem Effekt ist die Gabe von Noradrenalin die Therapie der Wahl. Dopamin sollte man angesichts des ohnehin schon erhöhten Risikos für Arrythmien vermeiden.

Hypertonie. Eine Hypertonie kommt bei Intoxikierten relativ häufig vor, insbesondere nach der Einnahme sympathomimetisch oder anticholinerg wirksamer Substanzen. Sofern zusätzlich Zeichen der Agitation vorliegen, setzt man zur Therapie in erster Linie unspezifische Sedativa ein (Benzodiazepine). Bei Zeichen einer Endorganschädigung sollte hingegen eine antihypertensive Therapie eingeleitet werden (Kalziumantagonisten, periphere $\alpha$-Blocker, ggf. Nitroprussid). Wichtig: Bei einer vermuteten symptomatischen Kokaineinnahme sollte man Betablocker nur dann einsetzen, wenn zuvor bereits eine vasodilatierende Substanz gegeben wurde. Kokain kann sonst über seinen $\alpha$-konstringierenden Effekt schwere Hypertonien und Koronarspasmen auslösen. Bei asymptomatischen Patienten sind Betablocker hingegen nicht kontraindiziert $[18,19]$.

Tachykarde Herzrhythmusstörungen. Die Erstlinientherapie bei ventrikulären Tachykardien oder Patienten mit einem verbreiterten QRS-Komplex ist die Gabe von Natriumbikarbonat ( $1-2 \mathrm{mmol} / \mathrm{kgKG}$ als Bolus). Damit unterscheidet sich das Vorgehen substanziell von der üblichen intensivmedizinischen Klientel, bei der Bikarbonatinfusionen selten indiziert sind. Der Grund ist, dass man bei Hinweisen auf Breitkomplextachykardien im Kontext einer Intoxikation immer an die Möglichkeit einer Natriumkanalblockade denken muss. Klassische Vertreter sind die trizyklischen Antidepressiva. Die Gabe von Natriumbikarbonat „antagonisiert“ diese Effekte. Eine Kontrolle der Wirksamkeit erhält man durch eine wiederholte EKG-Analyse, wobei sich die QRS-Dauer reduzieren sollte. Bei erfolgreicher Therapie schließt man kontinuierliche Infusion mit isotoner Bikarbonatlösung an. Der pH-Wert darf jedoch nicht über 7,55 ansteigen, da die Alkalisierung und damit einhergehende Elektrolytverschiebungen (Hypokaliämie!) zu einer Vasokonstriktion (Koronarien!) und QTc-Zeitverlängerung führen.

Eine gleichzeitige Substitution von Kalium und auch Magnesium (2g Magnesiumsulfat i. v. als Bolus) ist in den meisten Fällen notwendig und sinnvoll.

Die Erstlinientherapie bei vergifteten Personen bei ventrikulären Tachykardien oder verbreitertem QRS-Komplex ist die Gabe von Natriumbikarbonat ( $1-2 \mathrm{mmol} / \mathrm{kgKG}$ als Bolus). Die gleichzeitige Gabe von Antiarrhythmika der Klassen IA, IC und III wird ausdrücklich nicht empfohlen.

Gegen Amiodaron spricht die mögliche Verlängerung der QTc-Zeit. Jedoch gibt es bislang unzureichende Daten für eine abschließende Beurteilung. Denkbar ist hingegen der Einsatz von Lidocain (IB-Antiarrhythmikum), das sich zudem auch bei Dysrhythmien durch Kokain eignet $[20,21]$. 


\section{Infobox 6}

Ergänzende spezifische Therapie bei Bradykardie durch Medikamente

Intoxikation mit Kalziumantagonisten (am gefährlichsten ist der Verapamiltyp):

- 1-5g Kalziumchlorid oder Kalziumglukonat alle $15 \mathrm{~min}$ bis zur Erholung

- hoch dosierte Insulintherapie ( $1 \mathrm{IE} / \mathrm{kgKG}$ als Bolus gefolgt von $0,1 \mathrm{IE} / \mathrm{kgKG} / \mathrm{h}$ ) mit gleichzeitiger Gabe von Glukose

- Glucagon (1 - $10 \mathrm{mg}$ i.v.)

- Phosphodiesterasehemmer
Intoxikation mit Betablockern:

- Glucagon (50-150 $\mu \mathrm{g} / \mathrm{kgKG}$ als Bolus, gefolgt von einer Infusion $1-5 \mathrm{mg} / \mathrm{h}$ )

- anschließend Betamimetika

Intoxikation mit Digitalis:

- spezifischer Fab-Antikörper bei durch Digitalis ausgelösten lebensbedrohlichen Tachy- oder Bradyarrhythmien

- Cave: Die Digoxintoxizität beinhaltet eine mäßige Ansprache auf eine Schrittmacherstimulation.
Bei Zeichen einer drohenden Torsade-de-PointesTachykardie und/oder einer QTc-Verlängerung (>500 ms) sollte man Magnesiumsulfat verabreichen ( 2 g i.v.) [22].

Bradykarde Herzrhythmusstörungen. Bradykardien in Verbindung mit einer Hypotonie behandelt man wie sonst üblich mit Atropin und ggf. einem passageren Schrittmacher. Zur Optimierung des Blutdrucks eignet sich am besten Noradrenalin.

Eine zielgerichtete, differenzierte Therapie beinhaltet je nach Toxin noch weitere therapeutische Möglichkeiten, wobei der Blutzuckerspiegel eine differenzialdiagnostische Hilfe sein kann (Infobox 6). Bei einer Intoxikation mit Betablockern findet man meist eine Hypoglykämie, bei Kalziumantagonisten jedoch eher eine Hyperglykämie.

Krampfanfälle. Krampfanfälle behandelt man am besten mit Benzodiazepinen. Phenytoin wird aufgrund seiner kardialen Wirkungen ausdrücklich nicht empfohlen. Ebenso sollte man andere Präparate, die die QTc-Zeit verlängern, nur zurückhaltend einsetzen (Carbamazepin, Valproat).

Bei Krampfanfällen mit spezifischer Ursache sollte man natürlich entsprechend behandeln, z. B. mit Pyridoxin bei Isoniazidintoxikation oder Glukose bei einer Hypoglykämie.
Psychomotorische Erregungszustände. Psychomotorische Erregungszustände sind nicht selten bei sympathomimetisch und anticholinerg wirkenden Substanzen. Vor allem erschweren sie den Zugang zum Patienten. Hilfreich und sinnvoll ist zunächst die Schaffung eines ruhigen Umfelds, das beruhigend auf den Patienten einwirkt. So sollte man ggf. die Alarmlautstärken reduzieren und grelles Licht vermeiden. Dies ist auf der Intensivstation oft nicht einfach.

Ergänzend sollte man eine medikamentöse Therapie durchführen. Agitierte Verhaltensstörungen und psychotische Episoden sind am besten mit Sedativa (Benzodiazepine, z. B. Lorazepam) und hochpotenten Neuroleptika (Haloperidol, sofern keine Kontraindikationen vorliegen) zu behandeln [23]. Eine Ausnahme ist die Agitation im Rahmen eines anticholinergen Syndroms, für das Physostigmin als Antidot bereitsteht [24].

\section{Giftelimination}

Ein Punkt der 5-Finger-Regel ist die Giftelimination (Infobox 1). Nach der Klinikeinweisung sollte man, soweit möglich, Maßnahmen ergreifen, die die toxische Wirkung der eingenommenen Substanz reduzieren. Im Wesentlichen sind dies die primäre und sekundäre Giftentfernung und die Verabreichung von Antidota (sofern verfügbar). Die Giftelimination ist stark abhängig davon, wie die Noxen aufgenommen wurden und um welche es sich handelt.

\section{- Primäre Giftentfernung}

Aktivkohle. Kohle absorbiert aufgrund seiner großen Oberfläche eine Reihe von Toxinen, vor allem solche mit einer verzögerten Aufnahme (Infobox 7). Zwar gilt auch für die Gabe von Aktivkohle, dass die Noxenaufnahme möglichst nicht länger als 1 Stunde zurückliegt, allerdings ist angesichts geringer Nebenwirkungen auch eine spätere Applikation sinnvoll, um den enterohepatischen Kreislauf zu durchbrechen und eine verspätete Noxenaufnahme durch die Depotwirkung der Medikamente zu verhindern. Wichtig ist, dass die Dosis ausreichend hoch gewählt wird. Empfohlen werden derzeit 0,5-1 g Kohle/kgKG, also etwa 400-800 ml Suspensionslösung ( $1 \mathrm{ml}$ Kohlesuspension enthält 0,15g Aktivkohle). Besonders wichtig ist die Gabe einer ausreichenden Aktivkohledosis bei Substanzen mit einer retardierten Galenik. 


\section{Infobox 7}

Giftentfernung mit Aktivkohle

Beispiele für Substanzen, die von Aktivkohle adsorbiert werden:

- Antimalariamittel

- Theophyllin

- Barbiturate

- trizyklische Antidepressiva

- Carbamazepin

Nicht sinnvoll ist Aktivkohle bei:

- Pestiziden und Insektiziden

- Alkoholen

- Säuren und Laugen

- Lithium

Die Gabe von Aktivkohle ist kontraindiziert, solange die Schutzreflexe unzureichend und die Atemwege nicht geschützt sind. Auch ist die Routineanwendung bei nichttoxischen Substanzen nicht indiziert.

Aktivkohle verhindert die Absorption und sollte frühzeitig (und ggf. nach 4 Stunden erneut) in ausreichender Dosis gegeben werden $(2 \times 400 \mathrm{ml}$ für $80 \mathrm{kgKG})$.

Magenspülung. Immer wieder taucht die Frage auf, ob eine Magenspülung zur primären Giftentfernung noch indiziert ist. Die Antwort ist relativ eindeutig: nein [25]. Grund hierfür ist das relativ hohe Risiko aufgrund potenzieller Komplikationen wie Aspiration oder Perforation. Einschränkend gibt es jedoch Ausnahmen. Eine Magenspülung ist möglicherweise in den folgenden Fällen zu erwägen:

- Bei lebensbedrohlicher Vergiftung und sicherer Kenntnis der Einnahme innerhalb der letzten $60 \mathrm{~min}$ - was aber so gut wie nie vorkommt, denn allein bis die Magenspülung vorbereitet ist, sind meist schon 60 min vergangen - und einem erfahrenen Behandler, der jedoch oft nicht zur Verfügung steht.

- Bei bereits intubierten Patienten (dann ggf. mit Endoskop und Endowasher). Insbesondere ältere Patienten und solche mit einer erhöhten Komorbidität (Diabetiker) haben eine zum Teil erhebliche Gastroparese, die es ermöglicht, zahlreiche der geschluckten Tabletten abzusaugen. Ein endoskopisches Vorgehen hat gegenüber der Magenspülung auch den Vorteil, dass man tatsächlich sieht, ob noch etwas im Magen verblieben ist.
Magenspülung und provoziertes Erbrechen sind weitgehend obsolet und sollten nicht mehr durchgeführt werden.

\section{- Sekundäre Giftentfernung}

Eine weitere Möglichkeit der Eliminationsbeschleunigung ist die sekundäre Giftentfernung. Hier sind in erster Linie die Urinalkalisierung und extrakorporale Verfahren (Hämodialyse) zu nennen.

Urinalkalisierung. Für die Urinalkalisierung gilt als sinnvolle Indikation weiterhin die Salizylatintoxikation. Sonst hat dieses Verfahren in der klinischen Toxikologie einen begrenzten Stellenwert. Dies ergibt sich unter anderem auch aus der Tatsache, dass renal eliminierbare Substanzen hervorragend durch Hämodialyse entfernt werden könnten.

Extrakorporale Verfahren. Extrakorporale Therapieverfahren wurden schon mannigfach in der Hoffnung eingesetzt, damit lebensbedrohlich verlaufende Intoxikationen positiv zu beeinflussen. Neben den klassischen Nierenersatztherapien Hämofiltration (CVVH) und Hämodialyse wurden unter anderem auch Versuche unternommen, mit Immunadsorption oder therapeutischen Plasmaaustauschtherapien (Plasmapheresen) Giftstoffe zu entfernen.

Für die Nierenersatztherapie (Hämodialyse) bieten sich bei klinischen Intoxikationen im Wesentlichen 3 Einsatzgebiete an:

- Eliminierung eines Toxins

- Korrektur von mit Vergiftungen einhergehenden oder diese prognostisch limitierenden Säure-Basenund Elektrolytstörungen

- Therapie eines mit der Intoxikation einhergehenden Nierenversagens

Die Hämodialyse bietet sich ideal an zur Elimination von wasserlöslichen, kleinmolekularen Substanzen mit niedrigem Verteilungsvolumen und niedriger Proteinbindung. Hervorragend zu eliminieren sind dementsprechend die toxischen Alkohole Methanol und Ethylenglykol, ferner Salizylate, Lithium und Metformin. Ungeachtet dessen wurde in letzter Zeit für zahlreiche weitere Substanzen gezeigt, dass die Hämodialyse (evtl. als verlängerte Therapieform über viele Stunden, „extended daily dialysis“) in der Lage ist, auch vorwiegend proteingebundene Substanzen zu entfernen. So konnten unter anderem Carbamazepin, Valproat, Paraquat, Methotrexat, Thallium, Diphenhydramin, Theophyllin und Amitriptylin in wesentlichen Mengen eliminiert 
werden, obwohl deren pharmakokinetische Daten dies primär nicht vermuten lassen. Dies kann man unter anderem dadurch erklären, dass bei einer gesättigten Proteinbindung ein beträchtlicher Teil des Toxins als freie (und ggf. biologisch wirksame) Substanz zirkuliert, der dann aber durch Dialyse entfernt werden kann.

Pharmakokinetik ist nicht Toxikokinetik.

Zudem bieten sich nicht zuletzt aufgrund neuer, hochpermeabler Dialysemembranen, die auch deutlich größere Moleküle entfernen können, neue Indikationsspektren an. Hierzu ist mitunter eine Literaturrecherche hilfreich (Infobox 8).

Die Korrektur schwerer metabolischer Azidosen und lebensbedrohlicher Elektrolytstörungen sind eigentlich klassische Indikationen für eine Dialyse. Bei schweren Azidosen und drohender respiratorischer Erschöpfung kann die damit einhergehende Korrektur häufig eine Intubation verhindern. Insofern ist es eigentlich erstaunlich, dass diesbezüglich die Dialyse häufig erst verzögert eingesetzt wird. Gerade bei Intoxikationen mit Natriumkanalblockern (z. B. trizyklische Antidepressiva) bietet sich die Möglichkeit einer (isovolämischen!) Korrektur der Azidose und die gleichzeitige Optimierung des Natriums an (über ein hohes Natriumdialysat bis zu $150 \mathrm{mmol} / \mathrm{l}$ ). Bei Intoxikationen mit Methanol kommt es beispielsweise zu einer deut-
Infobox 8

Informationen zur Giftelimination durch extrakorporale Verfahren

- EXTRIP (http://extrip-workgroup.org)

- TOXNET (http://toxnet.nlm.nih.gov)

- Pubmed (www.pubmed.com)

lich schnelleren Entfernung von Methanol und auch der Abbauprodukte Formaldehyd und Ameisensäure (Infobox 9). Die einhergehende Azidose und Elektrolytstörungen wurden mit diesem sicheren Verfahren korrigiert und Morbidität, Mortalität und die Dauer des Krankenhausaufenthalts erheblich reduziert.

\section{Antidota}

„Behandele erst den Patienten, nicht das Gift“ ist prinzipiell der Eckpfeiler der Therapie bei Vergiftungen. Bei genauer Kenntnis der Noxe ist jedoch die Gabe eines spezifischen Antidots lebensrettend. Die Behandlung mit einem Antidot ist die ideale Therapie einer Vergiftung, da die Wirkung eines Gifts rasch neutralisiert oder in seiner Wirkung zumindest erheblich abschwächt wird (Tab. 1). Das Antidot selbst sollte natürlich keine toxischen Eigenschaften haben, was allerdings nur für Fomepizol (bei Ethylenglykolvergiftung) und Acetylcystein (bei Paracetamolvergiftung) weitgehend zutrifft (sofern keine allergischen Reaktio-

\section{Infobox 9}

Therapie bei gesicherter oder vermuteter Intoxikation mit Methanol oder Ethylenglykol

\section{Indikation}

Verdächtig auf eine Intoxikation mit Methanol oder Ethylenglykol ist eine metabolische Azidose mit hoher Anionenlücke. Therapieeinleitung, wenn folgende Kriterien erfüllt sind:

- dokumentierte Ingestion toxischer Mengen von Ethylenglykol oder Methanol und osmotische Lücke> 10 mOsm/l oder

- erhöhter Plasmaspiegel: Ethylenglykol> $20 \mathrm{mg} / \mathrm{dl}(3,2 \mathrm{mmol} / \mathrm{l})$ bzw. Methanol> $20 \mathrm{mg} / \mathrm{dl}(6,2 \mathrm{mmol} / \mathrm{l})$

oder

- vermutete Ingestion toxischer Mengen von Ethylenglykol oder Methanol
- metabolische Azidose (Bikarbonat<20 $\mathrm{mmol} / \mathrm{l}$, arterieller $\mathrm{pH}$-Wert $<7,3$ )

- osmotische Lücke>10 mOsm/l

- Nachweis von Oxalatkristallen im Urin (Ethylenglykol)

Therapie

- Initialdosis Fomepizol 15 mg/kgKG, danach $10-15 \mathrm{mg} / \mathrm{kgKG}$ alle 12 Stunden

- Hämodialyse bei schwerem Verlauf (Sehstörungen, Zeichen einer Nierenfunktionseinschränkung, hoher Spiegel)

Vorteile von Fomepizol:

- bereits bei geringen Serum-Konzentrationen wirksam
- minimale Nebenwirkungen

- kein Einfluss auf die mentale Aktivität, erleichtert die Beurteilung des klinischen Verlaufs

- Serum-Osmolalität wird nicht beeinflusst

Nachteile von Fomepizol:

- nicht überall sofort zum Einsatz vorhanden

- hoher Preis

- in Deutschland nur für Ethylenglykol zugelassen 


\section{Tabelle 1}

Klinisch wichtige Antidota.

\begin{tabular}{|l|l|}
\hline Antidot & Intoxikation \\
\hline $\begin{array}{l}\text { Hydroxocobalamin und } \\
\text { 4-Dimethylaminophenol } \\
\text { (4-DMAP) }\end{array}$ & Zyanidintoxikation \\
\hline Sauerstoff & Kohlenmonoxid, Rauchgas \\
\hline Fomepizol & Ethylenglykol \\
\hline Fomepizol und Ethanol & Methanol \\
\hline Glucagon & $\begin{array}{l}\text { Betablocker, Kalziumantago- } \\
\text { nisten }\end{array}$ \\
\hline $\begin{array}{l}\text { Digitalisantitoxin } \\
\text { Fab-Antikörper }\end{array}$ & \begin{tabular}{l} 
Digitalis \\
\hline Atropin
\end{tabular} \\
\hline Physostigmin & $\begin{array}{l}\text { Parasympathikomimetika, } \\
\text { Organophosphate und } \\
\text { Carbamate }\end{array}$ \\
\hline Methylenblau & Benzodiazepine \\
\hline Lipid-Infusion & (cave: Krampfneigung steigt) \\
\hline Naloxon & Paracetamol \\
\hline & Opiate \\
\hline
\end{tabular}

nen auftreten). Die Wirkung eines Antidots kann auf unterschiedlichen Effekten beruhen:

- Verhinderung der Absorption

- direkte Bindung oder Neutralisierung des Gifts

(z. B. Digitalisantikörper)

- Antagonisierung der Endorganwirkung (Naloxon, Atropin)

- Verhinderung der Bildung toxischer Metaboliten (Fomepizol)

Wie immer in der klinischen Medizin muss man das Nutzen/Risiko-Verhältnis abwägen, denn der gewünschte therapeutische Vorteil bei der Anwendung eines Antidots sollte natürlich die potenziellen Risiken überwiegen. Vorsicht ist z.B. geboten bei der Gabe von Flumazenil bei vermuteter Benzodiazepinintoxikation:

\section{Kernaussagen}

- Die primäre Therapie bei Intoxikationen gilt einer raschen Stabilisierung der Vitalparameter, wobei die Sicherung der Atemwege und die Überwachung des Herzrhythmus Priorität haben.

- Toxidrome leiten die klinische Beurteilung zur Abklärung der eingenommenen Substanzen.

- EKG und anschließende Überwachung zur Erfassung von Rhythmusproblemen sind zentraler Bestandteil der Einschätzung einer vital bedrohlichen Situation.

- Bei unklarer Intoxikation sollte man baldmöglichst Kontakt mit einer Giftinformationszentrale aufnehmen.

- Bei zahlreichen Intoxikationen sollte man neben der Behebung metabolischer Komplikationen auch eine Nierenersatztherapie zur Giftelimination in Betracht ziehen.

- Mischintoxikationen sind bei suizidaler Absicht häufig. Eine psychiatrische Beurteilung ist bei Suizidversuchen zwingend erforderlich.

Häufig werden zusammen mit Benzodiazepinen auch andere die Krampfschwelle senkende Medikamente eingenommen, sodass die Applikation von Flumazenil zu Krampfanfällen führen kann. Zudem ist zu beachten, dass die klinische Halbwertszeit des Antidots wesentlich kürzer sein kann als die des Toxins (z. B. Naloxon bei Opiatintoxikationen). Die wunderbar rasche Besserung des Patienten wiegt einen leicht in eine falsche Zuversicht, wenn man nicht an die eventuelle Notwendigkeit wiederholter Gaben denkt.

Leider gibt es nur für eine begrenzte Anzahl von Intoxikationen bekannte Antidota, die zudem auch nicht jede Klinik verfügbar hat. Es lohnt daher, den aktuellen Stand auszuloten und ggf. mit dem regionalen Giftinformationszentrum Rücksprache zu halten, in welcher Klinik welche Antidota verfügbar sind. Weitere Informationen über die Anwendungen von Antidota in der klinischen Toxikologie finden sich in guten Übersichtsreferaten [26].

Interessenkonflikt: Die Autoren geben an, dass kein Interessenkonflikt besteht. 


\section{Über die Autoren}

\section{Carsten Hafer}

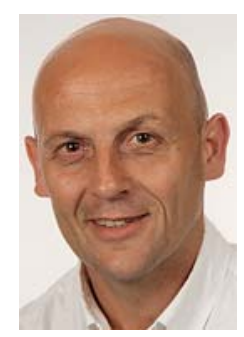

Dr. med. Jahrgang 1969. 1987-1994

Studium der Humanmedizin an der Medizinischen Hochschule Hannover. 1994-2002 Facharztausbildung zum Internisten. 2002 Facharzt für Innere Medizin, 2003 Facharzt für Nephrologie, 2011 Facharzt Internistische Intensivmedizin. Seit 2006 Oberarzt. 2011 Wechsel ans Nephrologische

Zentrum Niedersachsen. Schwerpunkte: extrakorporale Eliminationsverfahren und nephrologische Intensivtherapie, Säure-Basen- und Elektrolytstörungen.

\section{Jan T. Kielstein}

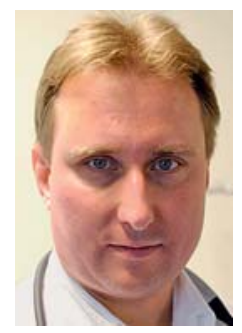

Prof. Dr. med. Jahrgang 1969. Medizinstudium an der Otto-von-Guericke Universität Magdeburg sowie an der Tufts Medical School in Boston und der Pritzker Medical School der University of Chicago. 1996-2004 Facharztausbildung zum Internisten (2004) und nachfolgend zum Nephrologen (2006). 2004-2007 Post-

doctoral Fellow am Falk Cardiovascular Research Center der Stanford. 2007 Habilitation, seither Oberarzt der Abteilung für Nieren- und Hochdruckerkrankungen und des Zentrums für Innere Medizin an der Medizinischen Hochschule Hannover. 2011 Ernennung zum außerplanmäßigen Professor. Schwerpunkte: Pathogenese der Atherosklerose bei Niereninsuffizienz sowie der Entwicklung und Evaluation extrakorporaler Verfahren in der Intensivmedizin.

\section{Korrespondenzadresse}

Dr. Carsten Hafer

Nephrologisches Zentrum Niedersachsen

Am Vogelsang 105

34346 Hann. Münden

E-Mail:c.hafer@comlink.org

\section{Literatur}

1 Camidge DR, Wood RJ, Bateman DN. The epidemiology of selfpoisoning in the UK. Br J Clin Pharmacol 2003; 56: 613-619

2 Wiegand T] et al. The Toxicology Investigators Consortium Case Registry - the 2011 experience. J Med Toxicol 2012; 8: 360-377

3 Benzer TI, Nejad SH, Flood JG. Case records of the Massachusetts General Hospital. Case 40-2013. A 36-year-old man with agitation and paranoia. N Engl J Med 2013; 369: 2536-2545

4 Giftinformationszentrum-Nord der Länder Bremen, Hamburg, Niedersachsen und Schleswig-Holstein (GIZ-Nord). Jahresbericht 2012. 2013 Im Internet: http://www.giz-nord.de/cms/ images/JaBe/2012/jabe12d.pdf Abruf 29.03.2014

5 Erickson TB, Thompson TM, Lu JJ. The approach to the patient with an unknown overdose. Emerg Med Clin North Am 2007; 25: $249-281$

6 Lin J et al. Repeated pulse of methylprednisolone and cyclophosphamide with continuous dexamethasone therapy for patients with severe paraquat poisoning. Crit Care Med 2006; 34: $368-373$

7 Wright N. An assessment of the unreliability of the history given by self-poisoned patients. Clin Toxicol 1980; 16: 381 384

8 Soslow AR. Acute drug overdose: one hospital's experience. Ann Emerg Med 1981; 10: 18-21

9 Yates C, Manini AF. Utility of the electrocardiogram in drug overdose and poisoning: theoretical considerations and clinical implications. Curr Cardiol Rev 2012; 8: 137-151

10 Niemann JT et al. Electrocardiographic criteria for tricyclic antidepressant cardiotoxicity. Am J Cardiol 1986; 57: 1154 1159

11 Prosser JM et al. Inaccuracy of ECG interpretations reported to the poison center. Ann Emerg Med 2011; 57: 122-127

12 Kraut JA, Kurtz I. Toxic alcohol ingestions: clinical features, diagnosis, and management. Clin J Am Soc Nephrol 2008; 3 : $208-225$

13 Sporer KA, Khayam-Bashi H. Acetaminophen and salicylate serum levels in patients with suicidal ingestion or altered mental status. Am J Emerg Med 1996; 14: 443-446

14 Lillsunde P. Analytical techniques for drug detection in oral fluid. Ther Drug Monit 2008; 30: 181 -187

15 Pemmerl S. Initial diagnosis and treatment for poisoning. Med Klin Intensivmed Notfmed 2013; 108: 459-464

16 Desel H. Toxicological analysis. Methodology, indication, and evaluation. Med Klin Intensivmed Notfmed 2013; 108: 471 475

17 Isbister GK et al. Aspiration pneumonitis in an overdose population: frequency, predictors, and outcomes. Crit Care Med 2004; 32: 88-93

18 Hollander JE. The management of cocaine-associated myocardial ischemia. N Engl J Med 1995; 333: 1267-1272

19 Lange RA et al. Potentiation of cocaine-induced coronary vasoconstriction by beta-adrenergic blockade. Ann Intern Med 1990; 112: 897-903

20 Albertson TE et al. TOX-ACLS: toxicologic-oriented advanced cardiac life support. Ann Emerg Med 2001; 37 (Suppl. 04): $78-90$ 
21 Hoffman RS. Treatment of patients with cocaine-induced arrhythmias: bringing the bench to the bedside. $\mathrm{Br} J$ Clin Pharmacol 2010; 69: 448-457

22 Drew BJ et al. Prevention of torsade de pointes in hospital settings: a scientific statement from the American Heart Association and the American College of Cardiology Foundation. Circulation 2010; 121: 1047-1060

23 Battaglia J et al. Haloperidol, lorazepam, or both for psychotic agitation? A multicenter, prospective, double-blind, emergency department study. Am J Emerg Med 1997; 15: 335 340
24 Burns MJ et al. A comparison of physostigmine and benzodiazepines for the treatment of anticholinergic poisoning. Ann Emerg Med 2000; 35: 374-381

25 Benson BE et al. Position paper update: gastric lavage for gastrointestinal decontamination. Clin Toxicol (Phila) 2013; 51: $140-146$

26 Hruby K. Antidotes in clinical toxicology. Med Klin Intensivmed Notfmed 2013; 108: 465-470 


\section{CME-Fragen}

\section{CME.thieme.de}

\section{CME-Teilnahme}

- Viel Erfolg bei lhrer CME-Teilnahme unter http://cme.thieme.de

- Diese Fortbildungseinheit ist 12 Monate online für eine CME-Teilnahme verfügbar.

- Sollten Sie Fragen zur Online-Teilnahme haben, unter http://cme.thieme.de/hilfe finden Sie eine ausführliche Anleitung.

\section{1}

Welche Aussage zu einer Intoxikation mit Ethylenglykol ist richtig?
A Man kann erst einmal in Ruhe abwarten, ob der Serumspiegel erhöht ist.
B Ethanol kann man aus Kosten- und Verträglichkeitsgründen Fomepizol vorziehen.
C Blindheit ist eine klassische Folge.
D Eine Hämodialyse kann einen substanziellen Therapiebeitrag leisten.
E Die osmotische Lücke ist immer erhöht.

\section{2}

Bei welchen Vergiftungen ist eine Urinalkalisierung sinnvoll?
A Methanol
B Salizylate
C Carbamazepin
D Zyanide
E Omeprazol

3

Wann ist eine Magenspülung sinnvoll?

4

Bei welchen Vergiftungen bzw. vergiftungsbedingten Situationen sollte man keine Hämodialyse durchführen?

5

Welche Aussage zu Digitalisintoxikationen ist richtig?
A niemals
B wenn der Patient das wünscht
C bei Ingestion einer lebensbedrohlichen Substanz innerhalb der letzten $60 \mathrm{~min}$
D immer bei intubierten Patienten
E bei Alkoholintoxikation (auch zur pädagogischen Prävention)
A Methanol
B Ethylenglykol
C bei schwerer metabolischer Azidose
D Knollenblätterpilze
E bei Nierenfunktionsbeeinträchtigung (AKIN 2-3)

A Man sollte zunächst den Kaliumspiegel anheben, um die Kardiotoxizität zu senken.

B Die Gabe von Kalzium ist eine sinnvolle therapeutische Unterstützung.

C Fab-Antikörper sind immer indiziert.

D Ggf. auch wiederholte EKG-Analysen sind sinnvoll.

E Ein passagerer Schrittmachereinsatz löst meist die wesentlichen Probleme. 


\section{CME-Fragen Therpie alutuer ntoxoxationen}

\section{6}

Welches ist keine Indikation zu einer intensivmedizinischen Überwachung im Rahmen einer Intoxikation?
A höhergradige AV-Blockierung
B respiratorische Azidose mit einem $\mathrm{CO}_{2}$ von $60 \mathrm{mmHg}$
C Linksschenkelblock im Aufnahme-EKG
D zerebraler Krampfanfall
E Alkoholspiegel von 1,2 Promille

\section{7}

Bei welchen Substanzen ist die Gabe von Aktivkohlegabe nicht hilfreich?
A Alkohole
B trizyklische Antidepressiva
C Barbiturate
D Carbamazepin
E Kalziumantagonisten

8

Welche Aussage zu initialen EKG-Veränderungen ist falsch?
A Eine verlängerte QTc-Zeit geht mit einem erhöhten Risiko kardiovaskulärer Ereignisse einher.
B Bei unauffälligem Aufnahme-EKG ist eine Überwachung nicht notwendig.
C Natriumkanalblocker gehen mit einer Verlängerung des QRS-Komplexes einher.
D Kaliumkanalblocker verkürzen die QT-Zeit.
E Rhythmusstörungen sind bei Intoxikationen häufiger als Ischämien.

Welche Maßnahme zur Absorptionsminderung hat den höchsten Stellenwert?
A Aktivkohle
B Darmspülung
C Apomorphin
D Kochsalz zum Auslösen von Erbrechen
E Magenspülung

\section{0}

Was gehört nicht zu den Indikationen einer stationären psychiatrischen Aufnahme nach einem Suizidversuch?
A akute Suizidgefährdung oder Versorgungsnotwendigkeit nach einem Suizidversuch
B Unsicherheit bei der Einschätzung der Suizidgefahr
C keine tragfähige therapeutische Beziehung
D Weiterbestehen von Suizidalität trotz adäquater Initialbehandlung
E Wunsch des Betreuers für medizinische Angelegenheiten 